\title{
Oral nalmefene reduced relapse to heavy drinking and was well tolerated in patients with alcohol dependence
}

\author{
Mason BJ, Salvato FR, Williams LD, et al. A double-blind, placebo-controlled study of oral nalmefene for alcohol \\ dependence. Arch Gen Psychiatry 1999 Aug;56:719-24.
}

QUESTION: In patients with alcohol dependence is nalmefene effective and safe?

\section{Design}

Randomised (unclear allocation concealment*), blinded (patients and outcome assessors, ${ }^{*}$ ) placebo controlled trial with 12 weeks of follow up.

\section{Setting}

The Alcohol Disorders Research Clinic, Jackson Memorial Medical Center, Miami, Florida, USA.

\section{Patients}

105 outpatients (mean age 42 y, $68 \%$ men) with a $D S M$ III-R diagnosis of current alcohol dependence recruited through advertisements and press releases. Exclusion criteria included dependence on illicit drugs; current use of naltrexone, disulfiram, medication containing narcotics, or psychotropic medications; notable medical or psychiatric disorders; considerable hepatocellular injury; and pregnancy. Patients were abstinent for a mean of 2 weeks before randomisation.

\section{Intervention}

Patients were assigned to oral nalmefene at a dose of 20 $\mathrm{mg} /$ day (10 $\mathrm{mg}$ twice daily) or $80 \mathrm{mg} /$ day (40 $\mathrm{mg}$ twice daily) $(\mathrm{n}=70)$, or placebo $(\mathrm{n}=35)$. All patients received individual, manual guided, cognitive behavioural therapy (CTB), as used in project MATCH (Matching Alcohol Treatments to Client Heterogenetics).
Sources of funding: in part, National Institute on Alcohol abuse and Alcoholism; the study drugs were provided by Ivax Corporation, Miami,FL, USA.

For correspondence: Dr B J Masom

University of Miami, Department of Psychiatry, Alcohol Disorders Research Unit, $1400 \mathrm{NW}$ 10th Avenue, Suite 307A,

Miami, FL 33136,

USA. Fax +1305243 2599.

\section{Main outcome measures}

Rate of relapse to heavy drinking (defined as $\geqslant 6$ drinks consumed/d for men and $\geqslant 4$ drinks/d for women), percentage of days abstinent, and change in the number of standard drinks consumed each drinking day over the 12 week follow up period.

\section{Main results}

Outcomes did not differ between the 20 and $80 \mathrm{mg}$ dose nalmefene groups and therefore these groups were combined in the intention to treat analyses. At the first weekly study visit, fewer patients in the nalmefene group reported any heavy drinking days compared with those in the placebo group $(\mathrm{p}<0.02)$ (table). Fewer patients in

Nalmefene v placebo for alcohol dependence $\dagger$

\begin{tabular}{lllll} 
Outcomes & Nalmefene & Placebo & RRR (95\% CI) & NNT(CI) \\
$\begin{array}{l}\text { Report of any heavy } \\
\text { drinking days at } 1 \text { week }\end{array}$ & $16 \%$ & $34 \%$ & $54 \%(7$ to 77$)$ & 6 (3 to 59$)$ \\
\hline $\begin{array}{l}\text { Relapsed to heavy drinking } \\
\text { over 12 weeks }\end{array}$ & $37 \%$ & $59 \%$ & $38 \%(6$ to 59$)$ & 5 (3 to 38$)$ \\
\hline
\end{tabular}

†Abbreviations defined in glossary; RRR, NNT, and $\mathrm{Cl}$ calculated from data in article. the nalmefene group relapsed to heavy drinking over 12 weeks of treatment than those in the placebo group $(p<0.02)$ (table). No differences existed for percentage of abstinent days or mean number of drinks consumed each drinking day. No medically serious adverse drug experiences were reported.

\section{Conclusion}

Oral nalmefene reduced relapse to heavy drinking and was well tolerated in patients with alcohol dependence.

*See glossary.

\section{COMMENTARY}

The study by Mason et al supports the role of opioid antagonists in preventing alcoholic relapse, although not in maintaining complete abstinence. CBT was provided. Studies evaluating the effectiveness of the opioid antagonist naltrexone find it to be more effective in conjunction with CBT than with supportive treatment. ${ }^{1}$ Rather than emphasising the absoluteness of total abstinence and avoidance of high risk situations, CBT helps the patient to cope with those situations by teaching new skills, problem solving techniques, and survival steps to follow if a drink is taken. Volpicelli $e t$ al found that naltrexone prevented relapse more often in patients who had drunk at least some alcohol. ${ }^{2}$ Sinclair proposed that opioid antagonists assist by enabling gradual extinction of the rewarding effects of alcohol. ${ }^{3}$ In the study by Mason et al nalmefene is seen to be effective in the first week; repeated drinking while on the drug is not needed for at least some effect to emerge.

Poor compliance with naltrexone prevents its benefits from emerging. ${ }^{4}$ Mason et al measured compliance by providing tablets in containers with microelectronic recorders, which may have helped them to achieve such high compliance rates.

In this study we are not told what the patients' goals were (abstinence or "controlled drinking") or what the patients were told about the putative mechanism of the effect of nalmefene. For extrapolation to routine practice, it would be helpful to know this. Jonathan Chick, MA, MPhil, FRCPE, FRCPsych Royal Edinburgh Hospital Edinburgh, UK

1 O'Malley SS, Jaffe AJ, Chang G, et al. Naltrexone and coping skills therapy for alcohol dependence. A controlled study. Arch Gen Psychiatry 1992;49:881-7.

2 Volpicelli JR, Alterman AI, Hayashida M, et al. Naltrexone in the treatment of alcohol dependence. Arch Gen Psychiatry 1992;49:876-80.

3 Sinclair JD. New treatment options for substance abuse from a public health viewpoint. Ann Med 1998;30:406-11.

4 Volpicelli JR, Rhines KC, Rhines JS, et al. Naltrexone and alcohol dependence. Role of subject compliance. Arch Gen Psychiatry 1997;54:737-42. 